\title{
Physiotherapy in the rehabilitation of sledge dogs
}

\author{
Liliya Trudova*, Anatoliy Stekolnikov, Alexandr Smolin, Anastasiya Bluzma, and Elizaveta \\ Titova \\ University of Veterinary Medicine, 196084 city of Saint Petersburg, Russian Federation
}

\begin{abstract}
The article deals with the current problems of rehabilitation of dogs participating in sledding competitions. The analysis of sports injuries among sled dogs was carried out, the main types of injuries were established, the general clinical condition of injured dogs was monitored, a clinical blood test was performed, the indicators of uric acid in the blood serum were determined, the main changes in the thermographic parameters of muscle groups were established before physiotherapy and after a course of restorative massage and low-frequency magnetic therapy. As a result of the conducted studies, it was found that the main injuries of sledge dogs were caused by violations associated with improper equipment and exterior deficiencies in the distal parts of the limbs, which was reflected in the overstrain of the shoulder girdle muscles. The use of massage and lowfrequency magnetic therapy contributed to the rapid recovery of the dogs' athletic form.
\end{abstract}

\section{Introduction}

The relevance of the chosen topic is determined by the fact that dog sleds are still a popular mode of transport among the indigenous peoples of the North. In addition, it is now a developing sports discipline, the geography of which is growing every year, involving an increasing number of people.

The organization of the training process in sled god racing includes a whole range of activities, which consists of checking the condition of the equipment and sled outfit; a balanced diet of sports dogs, taking into account their increased calorie needs during intense loads; monitoring of the physical condition of dogs and regular scheduled distributed speed loads.

Given that in sled dog sports, as in any other type of sports competitions, there are severe restrictions on the use of medicines for injuries, much attention is paid to the development of programs for injuries prevention and the use of environmentally friendly methods that do not cause addiction and the development of medicinal diseases. In this regard, physical therapy based on factors of physical nature deserves special attention [1].

Massage has a variety of effects on the body as a whole. Under the influence of massage movements, the stage of mechanical energy transformation into the energy of a nerve

\footnotetext{
${ }^{*}$ Corresponding author: mega.trudova@mail.ru
} 
impulse occurs, which gives rise to a complex chain of reflex reactions [2]. This method allows to affect the structures of the musculoskeletal system without drugs, restoring the full volume of lost movements [3,4].

Low-frequency magnetic therapy - the use of magnetic fields of various shapes, the frequency of which lies in the range of 1-200 Hz. The most sensitive to the action of this factor are the nervous, hematopoietic and endocrine systems. As a result of local exposure to a low-frequency magnetic field, the conduction velocity of potentials along nerve conductors increases, perineural edema decreases, increased vascular tone decreases, local blood flow is activated, blood viscosity decreases, and thus tissue nutrition improves[5].

The physiotherapy methods used in the work have a proven effective impact in many types of pathologies. Given the working value of the animals, this study aims to reduce the overall injury rate of sled dogs.

The purpose of the study was to evaluate the effectiveness of the use of physical therapy in sled dogs during the recovery period for injury prevention. Research tasks:

1. Consideration of the main causes of injuries in sled dogs;

2. Comprehensive diagnosis of sports injuries in sled dogs;

3. Physiotherapy for the prevention of sports injuries in sled dogs.

\section{Material and methods}

The research was conducted on the basis of a sled dog kennel. A total of 25 adult Husky sled dogs participating in sports races were examined. All the animals were kept in the same housing conditions, with the same feeding and training schedule. A general clinical examination was conducted in $5 \mathrm{dogs}$, and blood tests were taken to determine the concentrations of hemoglobin, red blood cells, and white blood cells, and to determine the level of uric acid. Immediately after the intensive training $(20 \mathrm{~km})$, a general clinical and orthopedic examination of sports dogs was conducted, as a result of which 10 dogs with signs of disorders of the shoulder girdle muscles were identified. Of these, 2 groups of animals were formed according to the principle of analogues, an experimental group of 5 dogs underwent rehabilitation using low-frequency magnetic therapy and restorative massage for 5 days, and 5 dogs from the control group were given rest from training for the duration of the study.

In both groups of dogs, blood was collected from the cephalic vein of the forearm immediately after intensive training and after 5 days. The hematological analyzer was used to determine the level of hemoglobin, the number of red blood cells and white blood cells; the level of uric acid in the blood serum was determined as an indicator of nitrogen metabolism intensity during exercise.

Thermography (real-time thermal imaging) was performed in dogs of both groups before and after training, as well as after a physical therapy session in dogs of the experimental group and in parallel in control dogs using the HT-02 infrared thermal imager (Fig. 1), a high-tech medical device that is successfully used in sports medicine for functional diagnostics of the body and monitoring of various methods of restorative treatment [6]. Thermography was performed on the shoulder girdle muscles (Fig. 2), since this is the most frequently injured area in sledge dog sports [7]. 


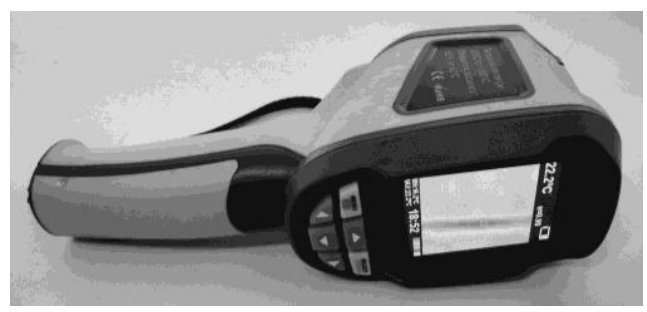

Fig.1. Thermal imager

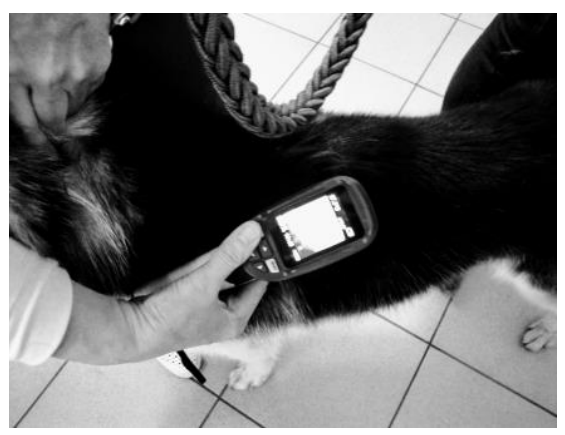

Fig. 2. The process of thermography in a dog

Immediately after intensive training, the dogs of the experimental group were exposed to the areas of spasmodic muscles with the low-frequency magnetotherapy device Magniter AMT-02 (RF) (Fig.3) with a magnetic induction value of 10 MT in the sinusoidal mode for 10 minutes (Fig. 4).

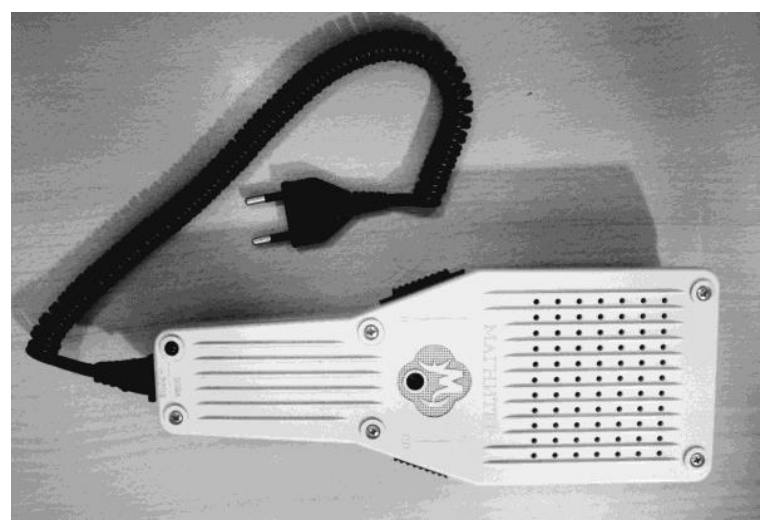

Fig. 3. Magniter AMT-02 device 
Fig. 4. Magnetic therapy procedure

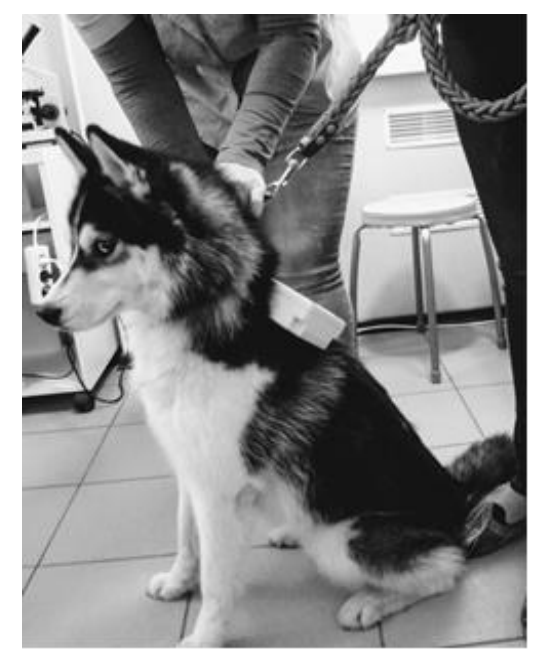

The device was placed in contact, without pressure. During the procedure, the dogs did not manifest anxiety.

Restorative massage (Fig.5) for 20 minutes, performed daily for 5 days, subject to certain rules:

1. Massage movements should be in the course of the lymphatic vessels and in the direction of the regional lymph nodes. The lymph nodes are not massaged;

2. Massage movements should not cause a painful and defensive reaction in the dog;

3. When performing a massage, the animal should be in such a position that the muscles of the massaged area are in a state of physiological rest or complete relaxation.

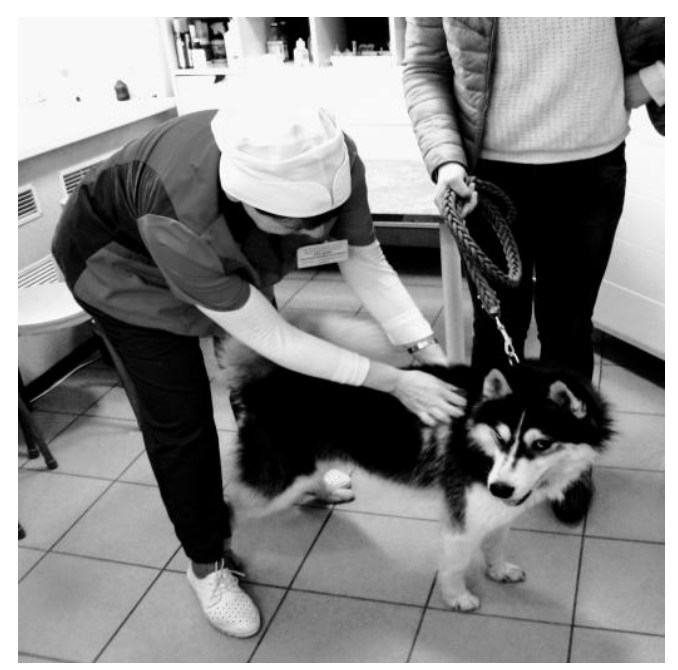

Fig. 5. Restorative massage

The massage program consisted of a double-sided muscles warm-up (infraspinatus, brachiocephalic, biceps), which was carried out in the form of kneading movements using the palms of both hands, while the muscle body moved back and forth using postural movements, with a gradual increase in impact intensity. Then a vibration massage of the 
stress zones was performed. At the same time, pressure was applied with the fingertips to the acupuncture points closest to the injured area. The movements are small, alternating between shallow and deep; 5 minutes at each point. The final stage of the massage program was relaxing soft movements directed along the hair growth.

\section{Research results}

As a result of physical examination after training, it was found that of all the observed sled dogs, $30 \%$ had signs of coordination myopathosis in the shoulder girdle, $10 \%$ of the dogs had injuries in the distal parts of the limbs (capellets, injuries of the claw cover and the area of the interdigital fold arch). Studying the causes of these pathologies, it was found that the muscle lesions were provoked by violations in the equipment. The riding harness is the most important piece of equipment for the effective work of the dog and reducing the risk of injury. Its correct position on the dog's body provides for the location of the upper chest straps strictly in the area of the withers, and in the studied dogs with shoulder girdle injuries, this element was located in the area of the shoulder blade, which caused a coordination violation in the work of the muscles. Our further studies included animals with myopathosis. In dogs with injuries of the distal extremities, the causes of damage were associated with external defects, namely, incorrect positioning of the limbs (weakness of the ligaments of the interdigital arch, violation of finger bones axis).

Simple coordination myopathosis was manifested by a violation of the rhythm and strength of contractions of the shoulder girdle muscles, an increase in the respiratory rate from $17.5 \pm 0.5$ to $28.7 \pm 0.3$ per minute, and a pulse from $90.8 \pm 0.8$ to $201.3 \pm 0.6$ per minute.

Thermography of dogs was carried out to determine the body temperature at rest and after exercise, the shoulder girdle muscles were selected as a sample, since they bear the greatest load. At rest, the temperature was in the range of $29.5 \pm 0.6^{\circ} \mathrm{C}$, after training in dogs of both groups it was increased by $36.2 \pm 0.3^{0} \mathrm{C}(\mathrm{p}<0.5)$, in the biceps area in all dogs there was an increased spasm.

The temperature of the shoulder girdle muscles in an hour after training in the control group decreased, but remained above normal and amounted to $32.3 \pm 0.7^{\circ} \mathrm{C}$, while the muscle spasm in the biceps area remained, and in the experimental group of dogs after 10 minutes of low-frequency magnetic therapy, the muscle spasm was completely eliminated and the temperature normalized to $30.1 \pm 0.3^{\circ} \mathrm{C}$.

Before intensive training, the indicators of hemoglobin level, concentrations of red blood cells and white blood cells in all the studied dogs were within the physiological norms, after training, there were no significant changes in these indicators. The level of uric acid in the blood serum, measured at rest, averaged $80.3+0.3 \mathrm{mmol} / \mathrm{l}$, which corresponds to the norm, after physical activity it increased to $110.2 \pm 0.4 \mathrm{mmol} / \mathrm{l}(\mathrm{p}<0.5)$.

After 5 days, all indicators returned to normal, in a repeated blood test in dogs in the control and experimental groups. When thermography of the biceps area in the dogs of the experimental group, the temperature was within the normal range, there was no muscle spasm, and in 2 dogs of the control group, the temperature remained elevated, the muscle spasm was not eliminated and some stiffness was observed in the movements.

\section{Results and discussion}

Based on the tasks related to the rehabilitation of sled dogs, the study found that violations in the equipment and exterior shortcomings of dogs were the cause of sports injuries (myopathosis of the shoulder girdle muscles and damage to the distal extremities). 
The resulting muscle spasm in the area of the proximal extremities leads to even more pronounced movement restrictions and deterioration of the distal extremities, and in the presence of external deficiencies in this area, muscle rigidity increases. The provision of rest to such athletic animals contributes to form recovery in the initial stages [1], but not always, as we noted in the course of our observations.

The use of a low-frequency magnetic therapy session in the sinusoidal mode of an alternating magnetic field with an induction of $10 \mathrm{MT}$, using the device "Magniter AMT02" for 10 minutes on the area of spasmodic muscles allowed to eliminate functional disorders, which was confirmed by the results of thermography. This effect is explained by the vasoactive, decongestant, analgesic, anti-stress effect of this physical factor in a nonthermal mode, which should be taken into account when exposed to the acute phase of damage. The biological effect of a low frequency alternating magnetic field is based on the stimulation of electrodynamic changes in tissue structures, which creates favorable conditions for the physical and chemical interactions of cells and the activation of their metabolism [8].

The course of restorative massage in the dogs of the experimental group in the future allowed them to completely restore their working form.

\section{Conclusions}

The use of low-frequency magnetic therapy and restorative massage made it possible to completely eliminate simple coordination myopathosis of the shoulder girdle muscles, while the rest provided to sled dogs of the control group did not contribute to the complete restoration of the working form of sled dogs.

Based on the results obtained, it is advisable to include a complex physiotherapy consisting of low-frequency magnetic therapy and restorative massage in the rehabilitation program of sled dogs. To prevent sports injuries, it is recommended to carefully select the equipment of the team and protective moccasins for dogs with incorrect positioning of the limbs, which have a greater risk of injury to the distal extremities.

\section{References}

1. V.V. Alecsandrov, A.I. Algazin, GEOTAR-Media, 50 (2013)

2. R. Couts, J. Katia, R. Ball, IN $28^{\text {th }}$ meeting of the orthopedic research society, 23 (1982)

3. C. Frank, W.H. Akeson, S.Woo, D. Amiel, R.D. Couts, Clinical orthopedics and related research, 185, 113 (1984)

4. R. Salter, D. Simmonds, B. Malcolm, J Bone Joint Surg Am., 62A, 1232 (1980)

5. V.E. Illarionov, T.V. Illarionova, Physiotherapy technologies of restorative medicine, 67 (2014)

6. A.V. Bokarev, A.A. Stekolnikov, M.A. Narusbaeva, V.E. Gorokhov, A.A. Imanbaev, Research Journal Of Pharmaceutical, Biological And Chemical Sciences, 10, 634 (2019)

7. L. Bogoslovskaya, Encyclopedia of the Arctic, 1, 501 (2005)

8. C.W. Smith, Conf. IEEE End. In Med. End Biol. Soc., 15-17, 176 (1984) 